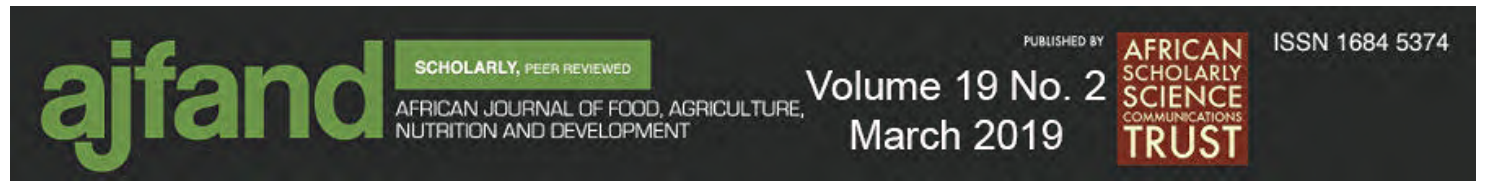

Afr. J. Food Agric. Nutr. Dev. 2019; 19(2): 14244-14260

DOI: 10.18697/ajfand.85.17435

\title{
LE KAWAL, UN CONDIMENT A BASE DE FEUILLES FERMENTEES DE SENNA OBTUSIFOLIA: TECHNOLOGIES ET VALEURS NUTRITIONNELLES
}

\author{
Abakar Idriss $\mathbf{L}^{1}$, Guira $\mathbf{F}^{1}$, Tapsoba $\mathbf{F}^{1}$, Zongo $\mathbf{C}^{1}$, \\ Abdoullahi Hissein $\mathbf{O}^{1,2,3}$, Tidjani $\mathbf{A}^{4}$ and A Savadogo ${ }^{1 *}$
}

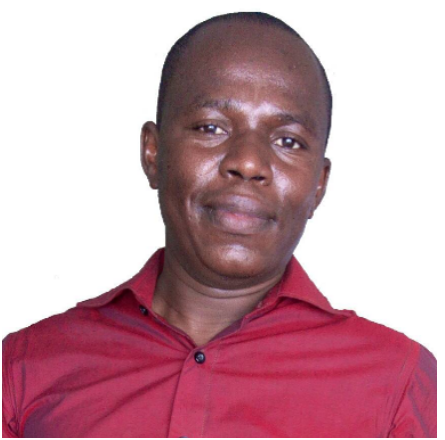

Abakar Idriss Lawane

*Contact auteur de correspondance: alysavadogo@gmail.com

${ }^{1}$ Laboratoire de Biochimie et Immunologie Appliquées (LaBIA/CRSBAN), Université Ouaga 1 Pr Joseph KI-ZERBO, 03 BP 7021 Ouagadougou 03

${ }^{2}$ Faculté des Sciences Exactes et Appliquées (FSEA), Laboratoire de Recherche sur les Substances Naturelles (LaRSN), Université de N'Djamena, BP 111.

${ }^{3}$ Laboratoire de Biotechnologie, des Sciences Alimentaires et Nutritionnelles (LaBSAN), Université de Ouagadougou, 03 BP 7021 Ouagadougou 03

${ }^{4}$ Faculté des Sciences de le Santé Humaine (FSSH), Laboratoire de Recherche en Sciences des Aliments et Nutrition (LaRSAN), Université de N'Djamena, BP 1117 


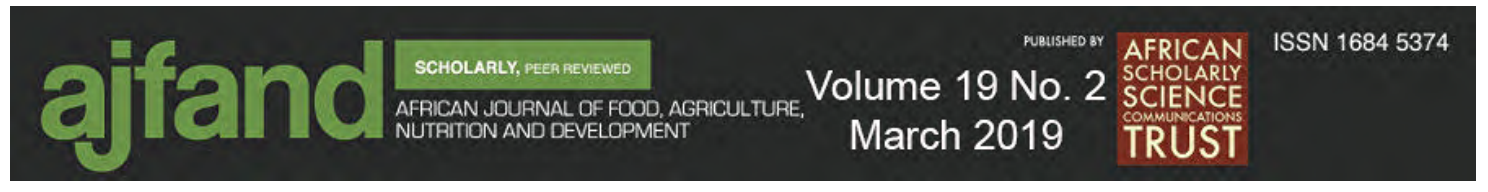

\title{
RÉSUMÉ
}

De nombreux aliments fermentés à base de légumes-feuilles sont consommés dans le monde, particulièrement en Afrique et en Asie. Ces aliments très répandus, représentent le régime de base en plus de leurs matières premières disponibles et constituent une part importante dans l'alimentation des populations locales. Le kawal obtenu par fermentation naturelle et alcaline des feuilles de Senna obtusifolia encore appelé Cassia obtusifolia, est un aliment très apprécié et largement consommé par les populations au Tchad et au Soudan. Les feuilles de S. obtusifolia occupent une place importante dans le système alimentaire de nombreuses communautés en Afrique. Elles sont riches en acides aminés essentiels et peuvent être considérées comme des produits d'intérêt nutritionnel de par leur valeur protéique. Le kawal contient des quantités appréciables de protéines et est utilisé comme substitut de viande et ou de poisson. Il est également riche en hydrates de carbone et en sels minéraux. La technologie de production du kawal reste traditionnelle avec des équipements rudimentaires et une fermentation incontrôlée. Cependant, cette technique de fermentation bien que traditionnelle permet l'élimination des facteurs antinutritionnels contenus dans les feuilles. Elle contribue aussi à l'amélioration de la valeur nutritionnelle et au développement de composés aromatiques tout en permettant d'augmenter la biodisponibilité des minéraux aboutissant ainsi à un produit qui permet de réduire les problèmes de carences en minéraux chez l'Homme. Les bactéries fermentaires du kawal, principalement celles des genres Bacillus et Lactobacillus du fait de leurs aptitudes probiotiques sont bénéfiques pour la santé humaine. La transformation de ce produit constitue un enjeu économique important en raison des revenus générés et contribue ainsi à la valorisation des ressources végétales et à la sécurité alimentaire des populations. Les technologies de la transformation du kawal n'étant pas bien maitrisées et variant d'une région à l'autre et ou d'une productrice à une autre il est donc indispensable de faire une synthèse sur les technologies de sa transformation et sa valeur nutritionnelle en vue de son amélioration.

Mots clés: Kawal, feuilles fermentées, Senna obtusifolia, technologies, valeurs nutritionnelles

\begin{abstract}
Many fermented foods containing vegetable leaves are mostly consumed in the world, particularly in Africa and Asia. These widespread foods represent the basic diet, in addition to their raw materials available, and constitute a significant share in the food of the local populations. Kawal, obtained by natural and alkaline fermentation of Senna obtusifolia also called Cassia obtusifolia leaves, is a much-appreciated food and largely consumed by the populations in Chad and Sudan. S. obtusifolia leaves play an important role in the food system for many communities in Africa. They are rich in essential amino acids and can be regarded as products of nutritional interest. The kawal contains considerable quantities of proteins and is used as a meat substitute. It is also rich in mineral salts and carbohydrates. The kawal technology production remains
\end{abstract}




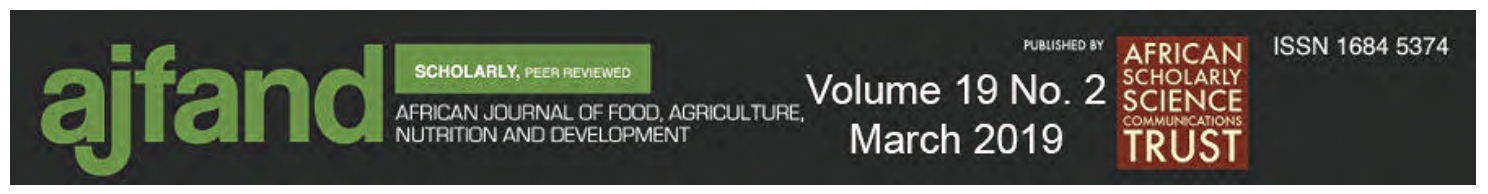

traditional with rudimentary equipment and an uncontrolled fermentation. However, this fermentation, although traditional, allows the elimination of the antinutritional factors contained in the leaves and also contributes to the improvement of the nutritional value and the development of aromatic compounds. It also makes it possible to increase the bioavailability of minerals and thus reduces the deficiency problems in humans. Bacillus and Lactobacillus genus in kawal play different beneficial roles to humans. Indeed, the kawal can be a source of probiotic microorganisms when the product is consumed without a stage of cooking after fermentation, thus keeping the probiotics alive and able to exert their beneficial effects for health. The transformation of this product constitutes a significant stake because of the generated incomes and thus contributes to the valorization of the forest resources and food safety. The technologies of the transformation of kawal not being well controlled and varying from one area to another, in the sight of an improvement, it is necessary to make a review on technologies of its transformation and on its nutritional value.

Key words: Kawal, fermented leaves, Senna obtusifolia, technologies, nutritional values 


\section{INTRODUCTION}

Utilisée depuis des siècles, la fermentation est la plus ancienne méthode de conservation alimentaire. Elle concerne une catégorie très vaste d'aliments : fruits, légumes, céréales et légumineuses, viandes, poissons, produits laitiers ou encore certaines boissons. Les aliments fermentés jouent un rôle important dans l'alimentation, la sécurité alimentaire et le bien-être social de millions de personnes à travers le monde. Ils contribuent pour 20 à $40 \%$ à l'alimentation dans le monde entier [1]. De nombreux aliments fermentés à base de légumes-feuilles sont consommés dans le monde, particulièrement en Afrique et en Asie, ces légumes représentant le régime de base en plus des matières premières disponibles. Les légumes-feuilles améliorent la qualité nutritionnelle des régimes alimentaires en raison de leur composition biochimique et leurs propriétés fonctionnelles [2,3]. Ils constituent une importante source de protéines, de vitamines, de fibres et de minéraux [4,5]. La plupart de ces légumes sont des espèces sauvages des forêts et utilisées par les communautés locales vivant dans différentes zones agroécologiques [6]. Parmi ces ressources forestières, Senna obtusifolia dont les feuilles fermentées sont appelées communément kawal est fortement consommé dans certains pays. C'est un produit fermenté considéré comme un substitut de viande, produit et consommé au Tchad, au Soudan et autres pays africains [7]. Il contient des quantités appréciables de protéines (20,2\% dans la feuille verte et environ $12,9 \%$ après fermentation), d'hydrates de carbone, et de sels minéraux [8,9]. Les techniques de transformation ou la fermentation utilisée permettent l'élimination des facteurs, antinutritionnels contenus dans les feuillets de Senna obtusifolia [9,10]. La fermentation contribue également à l'amélioration de la valeur nutritionnelle, au développement de composés aromatiques et la digestibilité du produit final [11]. Le kawal est très apprécié et largement consommé en Afrique Centrale. C'est pourquoi sa transformation constitue un enjeu important en raison des revenus générés pour les acteurs de la transformation contribuant ainsi à la valorisation des ressources forestières et à la sécurité alimentaire des populations. Dans le présent travail, nous faisons une synthèse sur les différentes technologies de transformation du kawal et de sa valeur nutritionnelle.

\section{Description et répartition géographique de Senna obtusifolia Description botanique}

Senna obtusifolia (L.) Irwin \& Barneby, encore appelée Cassia obtusifolia L. ou Cassia tora est une plante connue sous plusieurs noms vernaculaires : Kawal (au Tchad), Katre-nangouri en Moore et KriKri en Dioula (au Burkina Faso), Tasba (au Nigeria), casse fétide (en français), sicklepod (en Anglais), ebisu-gusa (au Japon), gyeolmyeongja (en Corée), fedegoso (en Portuguais) et sinameki (en Turkquie).

S. obtusifolia appartient à la sous-famille de Cesalpiniaceae et à la famille des Leguminosae [12]. C'est une plante herbacée annuelle ou vivace, arbrisseau atteignant 2 à 2,5 $\mathrm{m}$ de haut. Elle possède des feuilles alternes, imparipennées et portant 3 paires de folioles. Il y a un gradient de taille entre les folioles du sommet du rachis et celles de la base, les supérieures étant plus longues que les inférieures. Il y a 1 à 2 glandes en bâtonnet, situées sur le rachis au niveau des premières folioles. Le fruit est une gousse arquée de 10 à 15 centimètres de long. Senna obtusifolia est étroitement apparenté à 


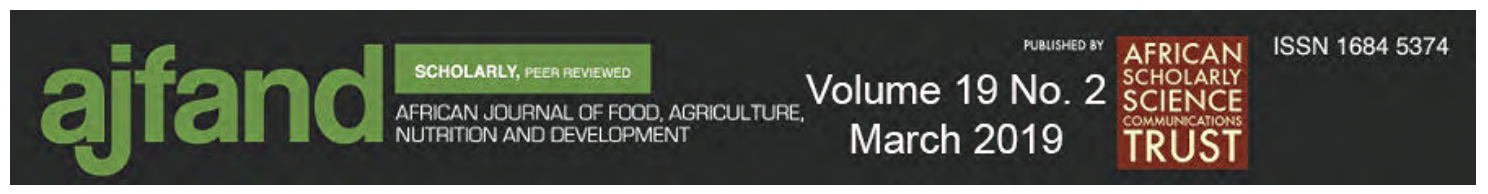

Senna tora L., mais cette dernière espèce se reconnaît à ses pédicelles plus courts. La présence en Afrique de Senna tora est douteuse et lorsqu'il est mentionné, il s'agit probablement de Senna obtusifolia [6].

\section{Répartition géographique}

Senna obtusifolia est probablement originaire de la région néotropicale $[12,13,14]$ mais elle s'est répandue dans toutes les régions tropicales et subtropicales du monde [15]

(Figure 1).

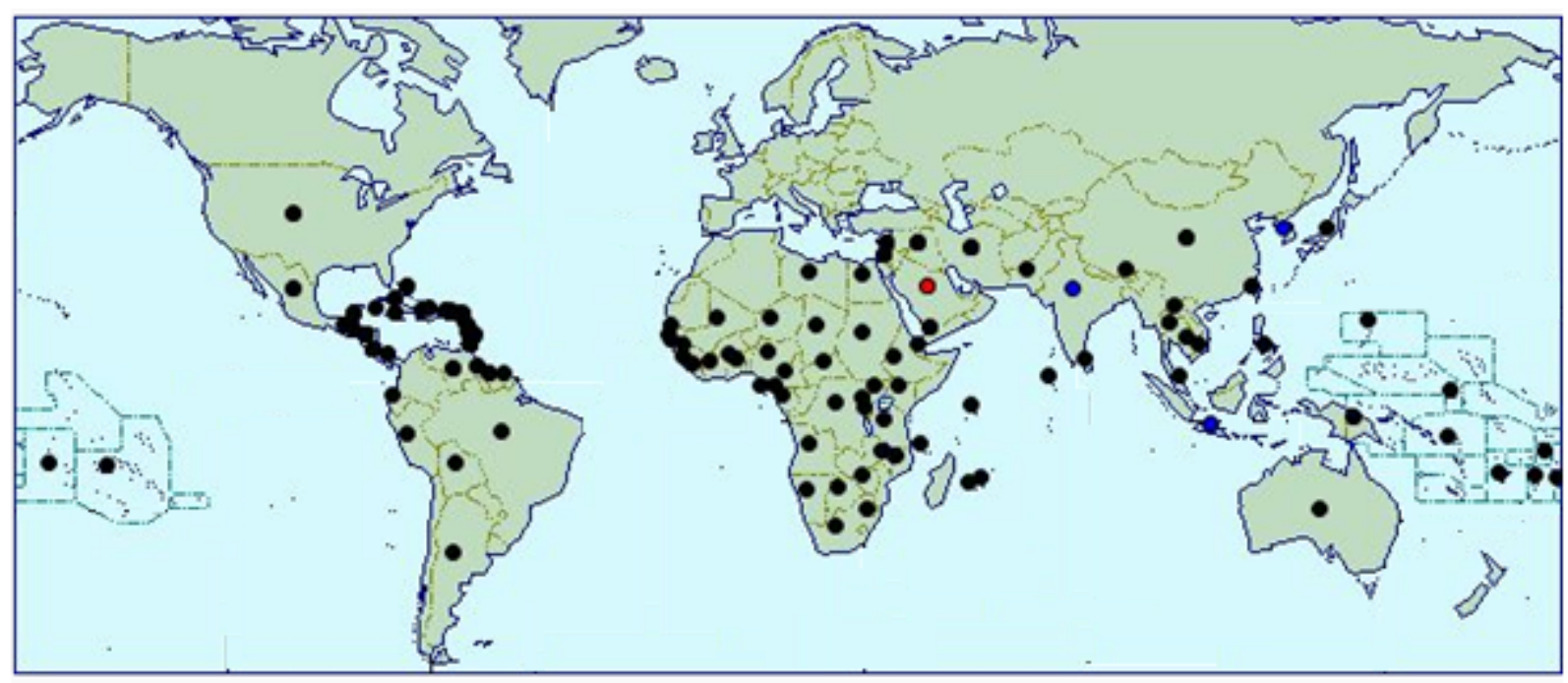

\section{Légende}

$\mathbf{O}=$ Présente, pas plus de détails
$=$ Répandue
$=$ Localisé

Figure 1: Répartition géographique de Senna obtusifolia dans le monde [15]

On trouve Senna obtusifolia dans toute l'Afrique tropicale, en Inde, au Sri Lanka, au Pakistan, en Malaisie, aux Philippines, en Indonésie, à la Papouasie-Nouvelle-Guinée, aux USA (Y compris Hawaii), au Mexique, aux Caraïbes, en Colombie, au Brésil, au Paraguay et en Argentine [12]. Il s'agit d'une plante annuelle, sauvage retrouvée sur les terres incultes surtout en saison pluvieuse. On la trouve le long des rivières et sur les rives des lacs, ainsi que sur les terres cultivées, jusqu'à $1700 \mathrm{~m}$ d'altitude [16]. Elle résiste sur les sols sableux et sablo-terreux qui ont une faible fertilité et une faible rétention d'eau.

\section{Les aliments fermentés à base de feuilles et de légumineuses}

De nombreux aliments fermentés sont consommés à travers le monde et chaque nation à ses propres types et/ou formes d'aliments fermentés, représentant le régime de base à partir des matières premières disponibles [17]. Ces aliments fermentés traditionnels sont très divers. Même si l'on se limite au continent Africain, on constate que les matières premières végétales sont également très variables et diversifiées et comprennent des racines, des légumes et des grains. Les aliments fermentés à base de légumineuses sont dominés par les bactéries du genre Bacillus qui réalisent une 
fermentation alcaline responsable du développement d'arômes et goûts très particuliers et associés souvent à des bactéries lactiques présentes en moindre proportion. Une large gamme de variétés de légumineuses est utilisée pour préparer des aliments fermentés en Afrique, en Asie du sud et en Amérique Latine mais aussi dans les îles du Pacifique. Certains aliments fermentés peuvent être fabriqués à partir de légumes graines ou à partir de légumes-feuilles et constituent une part importante dans l'alimentation des populations. Certains de ces produits sont consommés comme des plats de résistance, d'autres comme des appétissants ou de condiments utilisés dans les sauces.

En Afrique Centrale, les feuilles fermentées de manioc communément appelée ntobambadi sont utilisées pour la consommation humaine et très appréciées par son goût particulier et sa saveur et du fait de sa haute valeur nutritionnelle [18]. Par ailleurs, les feuilles de manioc sont riches en protéines, en minéraux et en vitamines. Il existe aussi une grande diversité de condiments issus de fermentations alcalines qui ont une importance alimentaire considérable dans plusieurs pays de l'Afrique de l'Ouest et de l'Afrique Centrale, notamment les produits obtenus par fermentation des graines de néré. Différents noms sont utilisés pour désigner ces produits identiques ou similaires. Par exemple, appelé soumbala au Burkina Faso, au Mali, en Côte d'Ivoire et en Guinée, il est connu sous le nom de dawadawa ou iru au Nigéria et au Nord du Ghana, nététu au Sénégal et afitin au Bénin [19]. En Asie, la fermentation des légumes-feuilles a été traditionnellement développée pour produire une large gamme de produits constitués essentiellement des feuilles de Brassica [20] qui sont connus sous différents noms, selon les procédés et les techniques utilisées traditionnellement. L'Inziangsang, le Gundruk et le Goyang sont obtenus respectivement à partir de feuilles de Brassicajuncea L. Czern, Brassicasp. et de Cardamine macrophylla. Le natto japonais est obtenus à partir de la fermentation du soja; le bikalga, l'ugba et l'ogiri sont généralement des produits obtenus à partir de la fermentation de graines d'Hibiscus sabdariffa, de fèves et de graines de melon respectivement [21,22,23]. Il est observé une augmentation du $\mathrm{pH}$ au cours de la fermentation de ces produits végétaux riches en protéines, due à l'activité protéolytique des microorganismes responsables de la fermentation qui dégradent les protéines de la matière première en acides aminés et en peptides. Une partie de ces acides aminés est dégradée pour produire des composés tels que le $\mathrm{NH}_{3}$ responsable de l'augmentation du $\mathrm{pH}$ [21]. La plupart des fermentations alcalines sont réalisées spontanément par des cultures de bactéries mixtes, dominées par Bacillus subtilis. Le tableau 1 décrit quelques aliments fermentés à base de feuilles et de légumineuses [24-30].

\section{Différentes utilisations de feuilles de $\boldsymbol{S}$. obtusifolia en alimentation}

Les feuilles de Senna obtusifolia sont parfois employées comme légume dans toute l'Afrique, en Inde, en Sri-Lanka et dans de nombreux pays du Sahel [31,32]. La plante est souvent cultivée dans les jardins familiaux pour cet usage dans plusieurs pays, dont le Sénégal, le Ghana, le Cameroun et l'Ethiopie. Elles sont considérées comme l'un des principaux légumes-feuilles indigènes du Sahel, et constitue une réserve alimentaire pour la population rurale pendant la saison pluvieuse et aussi a servi comme réserve alimentaire durant l'année de la famine au Soudan [10, 15, 33]. Les feuilles sont utilisées comme un complément minéral et vitaminique par certaines tribus au Kenya et au Sénégal [34]. En outre, les feuilles de $S$. obtusifolia, riches en protéines sont aussi 


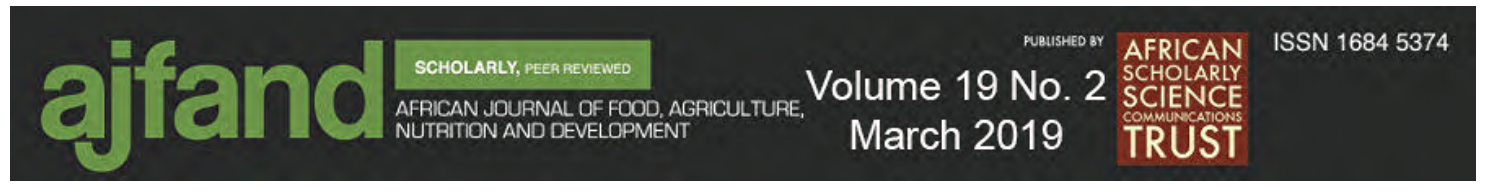

utilisées dans la formulation d'aliments pour la volaille [8,9]. Les feuilles fermentées de Senna obtusifolia sont utilisées comme un substitut de viande et condiment des sauces par de nombreuses communautés Tchadiennes et Soudanaises [35].

\section{Importance et valeurs nutritionnelles}

Les feuilles fermentées de Senna obtusifolia occupent une place importante dans la ration alimentaire de nombreuses communautés tchadiennes. Elles constituent pour les populations rurales une réserve alimentaire fiable et contribuent au renforcement des activités économiques des acteurs locaux. La forte teneur en protéines des feuilles fermentées de Senna obtusifolia en fait donc un aliment, qui a probablement protégé beaucoup d'enfants contre le kwashiorkor durant la famine [10]. Les feuilles de Senna obtusifolia, tout comme d'autres légumineuses constituent une source importante de nutriments. Ses constituants sont d'autant plus importants quand la feuille est fermentée $[8,9]$. Les valeurs nutritionnelles des feuilles de $S$. obtusifolia varient toutefois en fonction des auteurs et des variétés de Senna analysées (Tableau 2). Elles contiennent des proportions comparables en protéines, en acides aminés, en hydrates de carbone, en lipides, en énergie, en minéraux et autres nutriments [36,37].

Les valeurs des teneurs en protéines sont généralement comprises entre 15,52 et 30,20 $\%$ de matières sèches. Ces valeurs sont plus élevées que celles rapportées pour certains légumes-feuilles appartenant à d'autres espèces. Les protéines du kawal contiennent tous les acides aminés indispensables (l'isoleucine, la leucine, la lysine, la méthionine, la phénylalanine, la thréonine, le tryptophane, la valine) recommandés par l'Organisation des Nations Unies pour l'Alimentation et l'Agriculture [17]. Bien que sa teneur en acides aminés soufrés (la cystine et la méthionine) soit relativement faible, elle constitue une bonne source de lysine [37]. Le profil en acides aminés de la feuille fermentée est comparable à celui des légumineuses communes (Tableau 3). Ces concentrations en acides aminés essentiels complètent généralement les régimes à base de glucides fortement consommés par la population humaine en Afrique tels que les céréales et les racines, car ces dernières sont relativement pauvres en certains acides aminés essentiels. Le kawal contient des taux variables et importants de fibres brutes qui constituent l'un des composants majeurs de la feuille représentant entre 19,29 et 21, $66 \%$ de la matière sèche et une faible teneur en matières grasses $(3,97-4,8 \%$ de la matière sèche).

Les feuilles fermentées de $S$. obtusifolia contiennent également des quantités appréciables de minéraux (Tableau 4).

Le calcium et potassium sont les minéraux les plus abondants, avec un taux de 2933, 96 $\mathrm{mg} / 100 \mathrm{~g}$ et de $1924,97 \mathrm{mg} / 100 \mathrm{~g}$ respectivement. Cependant, on note de faibles teneurs en fer, en Magnésium, en Cuivre, en Zinc et en Phosphore [9].

\section{Teneur en composés organiques volatils}

Les principaux composés organiques issus de la métabolisation au cours de la fermentation du Kawal sont les acides gras volatils (AGV), les acides acétiques, les acides butyriques, les acides propioniques et les acides lactiques [8,37]. Les microorganismes du kawal ont un rôle essentiel dans la production de ces composés 


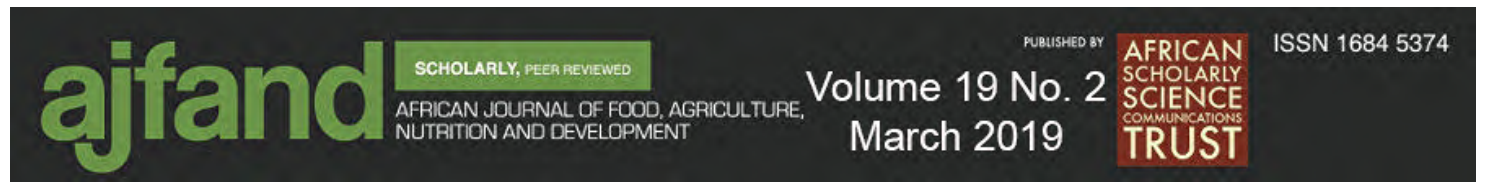

organiques et dans la dégradation des dérivés des protéines qui sont à l'origine de la saveur et de la flaveur [8,37]. Les travaux menés par Mbaiguinam et al. [37] ont montré que les extraits de feuilles fermentées de Senna obtusifolia sont constitués essentiellement d'acides aliphatiques. Parmi les acides aliphatiques, le 3méthylbutyrique représente $5,5 \%$ et le méthylbutyrique $2,5 \%$. Ces extraits contiennent aussi des composés phénoliques importants, le taux de p-méthylphénol est de $13 \%$ et p-éthylphénol de 17,21\%. La formation de certains alcools tels que le n-propanol, le nbutanol et l'éthanol a été détectée à la fin de la fermentation. Ces alcools se sont totalement volatilisés au cours de séchage [8].

\section{Microorganismes impliqués dans la fermentation des feuilles de $\boldsymbol{S}$. obtusifolia}

Les microorganismes responsables de la fermentation de feuilles de Senna obtusifolia sont très diversifiés et dominés par les bactéries du genre Bacillus avec comme espèces B. Subtilis, B. licheniformis, B. pumilus et B. amyloliquefaciens [37]. En plus des bactéries propioniques, il y a les bactéries lactiques appartenant au genre Lactobacillus, principalement Lactobacillus plantarum, comme principale espèce présente pendant la fermentation. Ces flores demeurent comme espèces dominantes durant la fermentation [8,37]. Les espèces Staphylococcus sciuri, Candida krusei et Saccharomyces sp. sont également présentes pendant la fermentation [8]. Cependant, les bactéries propioniques et les bactéries du genre Bacillus représentent les bactéries les plus largement retrouvées dans les feuilles fermentées séchées ou non [1,37], par leurs nombreuses activités protéolytiques, amylolytiques, lipasiques et phytasiques. Ces bactéries du genre Bacillus et les Bactéries lactiques présentent des intérêts technologiques et sanitaires. Les champignons sont responsables de la fermentation du soja pour produire le sufu et le tempeh.

\section{Techniques et procédés traditionnelles de fabrication du kawal}

Les étapes de production du kawal sont longues. Les principales opérations sont le pilage, la fermentation et le séchage [35].

\section{Sélection des feuilles}

Les feuilles sont récoltées à la fin de la saison des pluies lorsque la plante est bien développée. Ensuite, elles sont débarrassées de toutes matières étrangères et des diverses impuretés telles que les feuilles d'autres plantes, les vers, les insectes, les gousses et les fleurs de la plante elle-même.

\section{Pilage}

Les feuilles sélectionnées non lavées sont broyées par pilage au mortier-pilon en bois pour donner une pâte. Au cours de ce traitement mécanique, les feuilles subissent une transformation liée à la destruction du filament, la réduction et l'harmonisation de leur taille en vue d'obtenir une pâte et du jus. Ainsi, l'hydratation crée des conditions favorables à l'activité des enzymes microbiennes au cours de la fermentation. L'opération de pilage, assez pénible, est réalisée manuellement par pilage au mortier avec pilon en bois. 


\section{Fermentation}

La pâte obtenue est placée généralement dans une jarre en terre cuite et enterrée dans un endroit ombragé. La jarre est recouverte de pailles de sorgho. La fermentation est aussi longue comme celle d'autres produits fermentés d'origine végétale dans lesquels une fermentation alcaline est mise en œuvre. Cette fermentation dure généralement quinze (15) jours. La concentration en facteurs antinutritionnels diminue au cours de la fermentation $[9,10,11]$ par l'action enzymatique des microorganismes.

\section{Malaxage}

À un intervalle de 3 jours, la jarre est ouverte, les feuilles de sorgho sont enlevées et la pâte soigneusement homogénéisée manuellement. La pâte est ensuite remballée et la jarre couverte de nouvelles feuilles fraîches de sorgho. Il permet d'obtenir une pâte bien homogénéisée durant la fermentation.

\section{Moulage et séchage}

Après 11 à 15 jours, la masse est retirée, moulée en petites boules et séchée au soleil pendant 3 à 5 jours. Il consiste à déshydrater l'amande fraîche contenue dans la pâte fermentée de façon à réduire sa teneur en eau à moins de $12 \%$ [9,37]. Ce facteur limite la prolifération microbienne et ralentit les réactions chimiques et enzymatiques, ce qui assure la stabilité du produit et maintient sa qualité au cours du stockage. La pluie et le climat humide conduisent souvent à écourter l'opération. Pour que les produits soient séchés et surtout à moins de $12 \%$, il faut suffisamment de soleil, ce qui est souvent une contrainte majeure en saison pluvieuse. Un temps trop humide pendant le séchage conduit à l'obtention de produits de moindre qualité fréquemment contaminés par des microorganismes indésirables. Après le séchage, les kawals sont écrasés à l'aide d'un mortier afin d'obtenir une masse très fine et utilisés comme ingrédients ou condiments dans la sauce. Les produits finals sont conditionnés dans des sacs en puis stockés.

Les bonnes pratiques de stockage sont nécessaires pour empêcher la réhumidification de kawal et lutter contre les microorganismes d'altération ou pathogènes. Pour le stockage de longue durée, un séchage complémentaire est nécessaire pour empêcher le développement de ces germes indésirables. Parmi ces pratiques, on peut citer une toiture en bon état, une bonne ventilation, un plancher surélevé et un traitement contre les insectes et les ravageurs. Ainsi, le kawal réduit en poudre et très bien emballé est proposé directement aux consommateurs.

L'utilisation de la méthode "Hazard Analysis Critical Control Point» (Système d'analyse des risques-points critiques pour leur maîtrise (HACCP) en technologie du kawal s'appuyant solidement sur les Bonnes Pratiques de Fabrication (BPF) et Bonnes Pratiques d'Hygiène (BPH) et la mise en place d'un Plan de Maîtrise Sanitaire (PMS) devrait permettre d'assurer la maîtrise des dangers. Ces dangers, pouvant apparaître à tous les niveaux de la chaîne de production du Kawal. Cela permettrait de garantir la qualité nutritionnelle, hygiénique et sanitaire du produit [31] et de le rendre commercialement plus compétitif vis-à-vis des condiments importés. Ces procédés de transformation traditionnelle sont souvent réalisés dans des conditions insuffisantes de bonnes pratiques d'hygiène [37]. Il est donc important que tous ces procédés de 


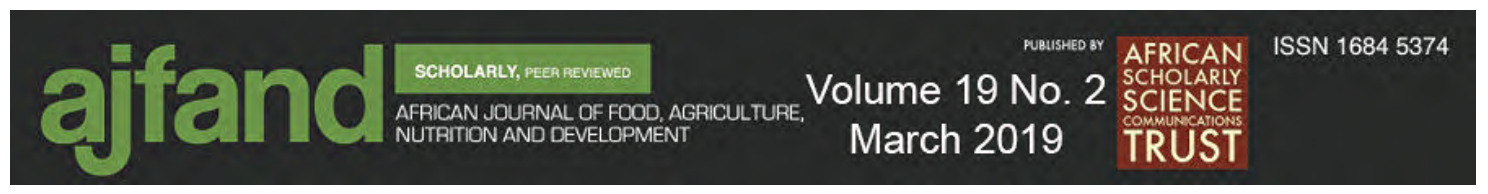

transformation traditionnelle se déroulent dans des conditions de sécurité sanitaire pour garantir la sécurité du consommateur. Les opérations de malaxage, de moulage et de séchage solaire au cours de la production du kawal sont des opérations critiques importantes à contrôler pour éviter les contaminations par de micro-organismes exogènes et ou les composés chimiques. Cela serait possible grâce au respect strict des bonnes pratiques d'hygiène et des bonnes pratiques de fabrication. En effet, il est important d'utiliser des micro-organismes à potentiel probiotique et métabolique [31] avec l'application Bonnes Pratiques d'Hygiène et des Bonnes Pratiques de Fabrication pour un kawal de meilleure qualité hygiénique et sanitaire.

\section{CONCLUSION}

Les feuilles fermentées de $S$. obtusifolia présentent une valeur nutritive considérable, les composés les plus importants étant les protéines, les sels minéraux et les fibres alimentaires. Elles sont particulièrement riches en acides aminés essentiels et elles ont une valeur énergétique importante. La fermentation de feuilles de S. obtusifolia permettrait l'élimination des facteurs antinutritionnels contenus dans les feuilles fraiches et contribue aussi à l'amélioration de la valeur nutritionnelle. Les bactéries du genre Bacillus et de Lactobacillus impliqués dans la fermentation du Kawal jouent différents rôles bénéfiques pour l'Homme et la qualité du produit final. Toutes ces qualités montrent leur importance pour la sécurité alimentaire. La fermentation contrôlée avec l'application des Bonnes Pratiques d'Hygiène et des Bonnes Pratiques de Fabrication rend la transformation plus efficace et plus profitable permettant ainsi d'obtenir des produits de qualité nutritive, hygiénique et organoleptique satisfaisante. 


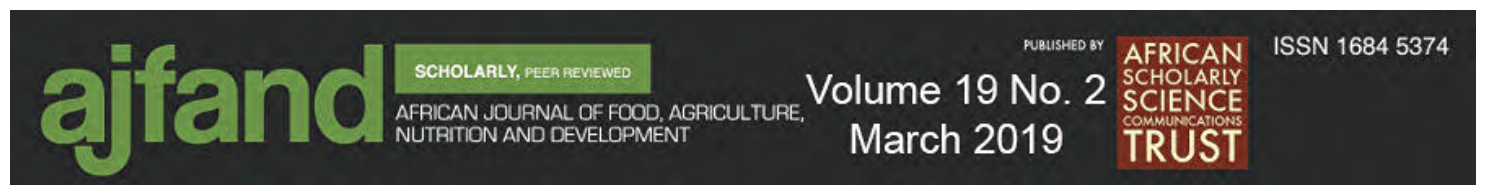

Tableau 1: Exemples d'aliments fermentés à base de feuilles et de légumineuses

\begin{tabular}{|c|c|c|c|}
\hline Matrice & Nom local du produit & Pays d'origine & Sources \\
\hline Feuilles de manioc & Ntobambodi & Congo & {$[18]$} \\
\hline $\begin{array}{l}\text { Feuilles de Brassica } \\
\text { carinata }\end{array}$ & kale & kenya & {$[25]$} \\
\hline Feuilles de Brassicajuncea & Inziangsang & Inde & {$[20]$} \\
\hline $\begin{array}{l}\text { Feuilles de Cardamine } \\
\text { macrophylla }\end{array}$ & Goyang & Inde & {$[26]$} \\
\hline Feuilles de Brassica $s p$ & Gundruk & Inde & {$[20]$} \\
\hline Graines de baobab & Maari & Burkina Faso & {$[27]$} \\
\hline \multirow[t]{4}{*}{ Graines de néré } & Soumbala & Burkina Faso & {$[21 ; 23]$} \\
\hline & Netetu & Sénégal & {$[33]$} \\
\hline & Afitin, Iru & Benin & {$[28]$} \\
\hline & Dawadawa (Iru) & Nigeria & [29] \\
\hline $\begin{array}{l}\text { Graines d'Hibiscus } \\
\text { sabdariffa }\end{array}$ & Bikalga & Burkina Faso & {$[21 ; 23]$} \\
\hline Prosopis africana & Okpehe & Nigeria & {$[25]$} \\
\hline \multirow[t]{3}{*}{ Graines de soja } & Soydawadawa & Ghana & {$[30]$} \\
\hline & Soyadawadawa & Nigeria & {$[21]$} \\
\hline & Natto & Japon & {$[21]$} \\
\hline
\end{tabular}




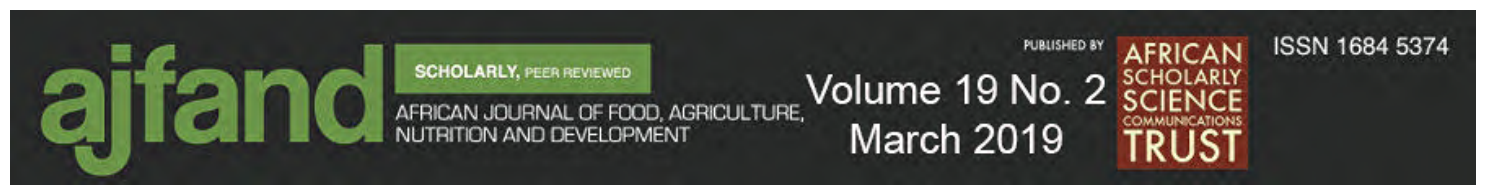

Tableau 2: Composition biochimique de feuilles fraiches et de feuilles fermentées de Senna obtusifolia

\begin{tabular}{|l|l|l|l|l|l|l|l|c|}
\hline Feuilles & \multicolumn{7}{|c|}{ Constituants (\%) } & \multirow{2}{*}{ Sources } \\
\cline { 2 - 9 } & Protéines & HC & Lipides & Fibres & Cendres & MS & VE kcal/100 g) & \\
\hline \multirow{5}{*}{ Fraiches } & 21,4 & 5,1 & 4,8 & 13,9 & 10,3 & ND & ND & {$[36]$} \\
\cline { 2 - 9 } & 21,87 & 28,88 & 3,97 & 24,18 & 12,08 & 93,93 & 268,85 & {$[9]$} \\
\cline { 2 - 9 } & 24,3 & ND & 2,5 & 13,5 & 12,6 & ND & ND & {$[8]$} \\
\hline \multirow{5}{*}{ Fermentées } & 26,2 & ND & 3,8 & 12,1 & 19,6 & ND & ND & {$[8]$} \\
\cline { 2 - 9 } & 30,20 & 22,68 & 4,12 & 21,66 & 18,74 & 92,50 & 232,88 & {$[9]$} \\
\cline { 2 - 9 } & 19,23 & ND & ND & ND & 18,73 & 91,29 & ND & {$[37]$} \\
\hline
\end{tabular}

HC : Hydrates de carbone ; MS : Matière sèche ; VE : Valeur énergétique ; ND : Non déterminé 


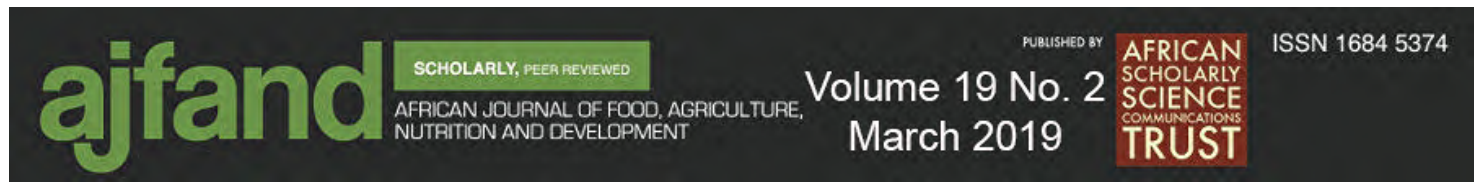

Tableau 3: Profil en acides aminés de feuilles fraiches et des feuilles fermentées de S. obtusifolia

\begin{tabular}{|c|c|c|c|c|}
\hline \multirow{3}{*}{ Acides aminés } & \multicolumn{4}{|c|}{ Sources } \\
\hline & \multicolumn{2}{|c|}{$[35]$} & \multicolumn{2}{|r|}{$[6]$} \\
\hline & $\begin{array}{c}\text { Feuilles } \\
\text { fraiches } \\
(\mathrm{mg} / 100 \mathrm{~g})\end{array}$ & $\begin{array}{c}\text { Feuilles } \\
\text { fermentées } \\
(\mathrm{mg} / 100 \mathrm{~g})\end{array}$ & $\begin{array}{c}\text { Feuilles } \\
\text { fraiches } \\
\left(\mathrm{g} 16 \mathrm{~g}^{-1} \mathrm{~N}\right)\end{array}$ & $\begin{array}{l}\text { Feuilles fermentées } \\
\qquad\left(\mathrm{g} 16 \mathrm{~g}^{-1} \mathrm{~N}\right)\end{array}$ \\
\hline \multicolumn{5}{|c|}{ Acides aminés essentiels } \\
\hline Thréonine & 980 & 522 & 6,2 & 3,3 \\
\hline Valine & 1606 & 1282 & 7,5 & 6,4 \\
\hline Méthionine & 11 & 18 & 2,1 & 1,5 \\
\hline Isoleucine & 1181 & 987 & 6,0 & 5,1 \\
\hline Leucine & 2234 & 1803 & 10,4 & 8,3 \\
\hline Phénylalanine & 1562 & 1022 & 6,8 & 5,4 \\
\hline Lysine & 1477 & 790 & 7,7 & 4,0 \\
\hline \multicolumn{5}{|c|}{ Acides aminés non essentiels } \\
\hline Histidine & 664 & 356 & 3,3 & 2,0 \\
\hline Arginine & 1314 & 601 & 7,2 & 4,0 \\
\hline Acide aspartique & 3786 & 1345 & 12,1 & 7,7 \\
\hline Sérine & 863 & 505 & 4,6 & 2,8 \\
\hline Proline & 1769 & 858 & 7,7 & 4,2 \\
\hline Glycine & 1428 & 1074 & 6,7 & 5,0 \\
\hline Cystine & 13 & 4 & 1,4 & 1,2 \\
\hline Tyrosine & 435 & 239 & 5,3 & 3,5 \\
\hline Alanine & 1595 & 1638 & 7,5 & 6,8 \\
\hline
\end{tabular}




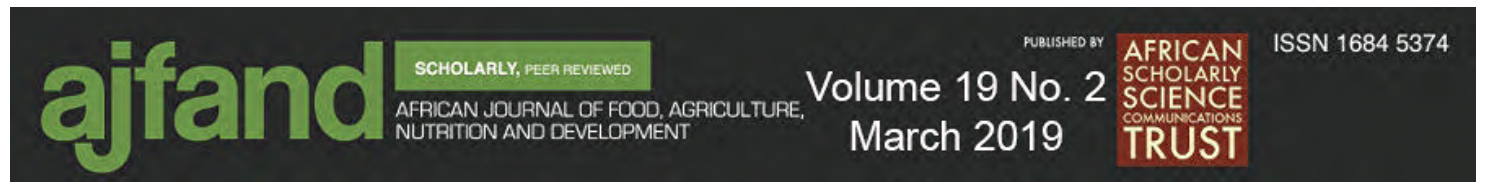

Tableau 4: Composition en éléments minéraux de feuilles fermentée de Senna obtusifolia

\begin{tabular}{|c|c|c|c|c|c|c|c|c|c|}
\hline \multicolumn{9}{|c|}{ Eléments minéraux (MS) } & \multirow{2}{*}{ Sources } \\
\hline $\mathrm{Ca}$ & $\mathrm{Mg}$ & $\mathrm{P}$ & $\mathrm{Fe}$ & $\mathrm{Zn}$ & $\mathrm{Mn}$ & $\mathrm{Na}$ & $\mathrm{Cu}$ & $\mathrm{K}$ & \\
\hline $\begin{array}{c}5973,95 \\
\mathrm{mg} / 100 \mathrm{~g}\end{array}$ & $\begin{array}{c}626,50 \\
\mathrm{mg} / 100 \mathrm{~g}\end{array}$ & $\begin{array}{c}593,35 \\
\mathrm{mg} / 100 \mathrm{~g}\end{array}$ & $\begin{array}{c}61,48 \mathrm{mg} / \\
100 \mathrm{~g}\end{array}$ & $\begin{array}{c}6,92 \\
\mathrm{mg} / 100 \mathrm{~g}\end{array}$ & $\begin{array}{c}14,79 \\
\mathrm{mg} / 100 \mathrm{~g}\end{array}$ & $\begin{array}{c}175,67 \\
\mathrm{mg} / 100 \mathrm{~g}\end{array}$ & $\begin{array}{c}5,03 \\
\mathrm{mg} / 100 \mathrm{~g}\end{array}$ & $\begin{array}{c}2054,05 \\
\mathrm{mg} / 100 \mathrm{~g}\end{array}$ & [9] \\
\hline $\begin{array}{l}27250 \\
\mathrm{mg} / \mathrm{kg}\end{array}$ & $\begin{array}{c}1490 \\
\mathrm{mg} / \mathrm{kg}\end{array}$ & $\begin{array}{c}2800 \\
\mathrm{mg} / \mathrm{kg}\end{array}$ & $\begin{array}{c}0,4 \\
\mathrm{mg} / \mathrm{kg}\end{array}$ & ND & ND & $\begin{array}{c}1310 \\
\mathrm{mg} / \mathrm{kg}\end{array}$ & ND & $\begin{array}{l}31500 \\
\mathrm{mg} / \mathrm{kg}\end{array}$ & {$[38]$} \\
\hline $4,13 \%$ & $0,42 \%$ & $0,28 \%$ & $82 \mathrm{mg} / \mathrm{kg}$ & $\begin{array}{c}84 \\
\mathrm{mg} / \mathrm{kg}\end{array}$ & $\begin{array}{c}112 \\
\mathrm{mg} / \mathrm{kg}\end{array}$ & ND & ND & ND & {$[8]$} \\
\hline
\end{tabular}

ND : Non déterminé 


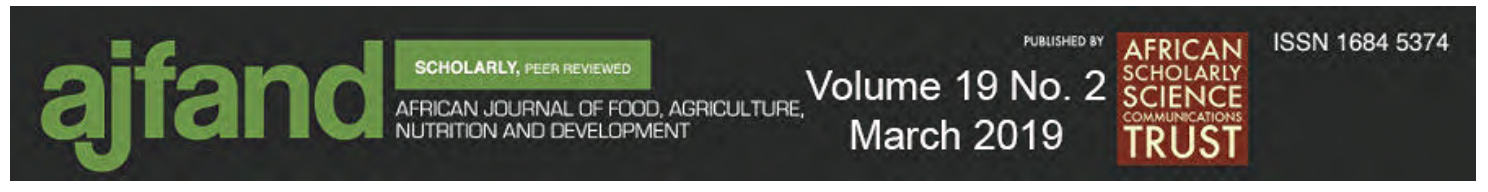

\section{RÉFÉRENCES}

1. Campbell-Platt G Fermented foods: a world perspective. Food Res. Int. 1994; 27: 253-257.

2. Dansi A, Adjatin A, Adoukonou-Sagbadja H, Adomou A, Falade V, Yedomonhan H, Akpagana $K$ and B De Foucault Traditional leafy vegetables in Benin: Folk nomenclature, species under threat and domestication. In Biodiversité des Légumes-feuilles Traditionnels Consommés au Bénin. Bibliothèque Nationale : Bénin, 2008: 173.

3. Tarnagda B, Tankoano A, Tapsoba F, Pane BS, Hissein OA, Djbrine AO and A Savadogo Évaluation des pratiques agricoles des légumes feuilles: le cas des utilisations des pesticides et des intrants chimiques sur les sites maraîchers de Ouagadougou, Burkina Faso. J. Appl. Biosci., 2017 ; 117(1) : 11658-11668.

4. Traoré K, Parkouda C, Savadogo A, Kamga $R$ and $Y$ Traoré Effect of processing methods on the nutritional content of three traditional vegetables leaves: Amaranth, black nightshade and jute mallow. Food Sci. Nut 2017; 5 (6): 1139-1144.

5. Ganiyu $\mathbf{O}$ Nutritional and safety evaluation of some tropical green leafy vegetables. J. Food Technol. 2005; 3: 389-392.

6. Grubben GJH and OA Denton Ressources végétales de l'Afrique tropicale 2 . Légumes. (Ttraduction de : Plant Resources of Tropical Africa 2. Vegetables. 2004). Fondation PROTA. Wageningen, Pays-Bas/BakhuysPublishers. Leiden. Pays- Bas/CTA.Wageningen, Pays-Bas, 2004 : 737.

7. Dirar HA Flavours and substitutes of plant origin: in The Indigenous Fermented Foods of the Sudan. UK. CAB Int\& Wallingford, 1993: 447.

8. Dirar HA, Harper DB and MA Collins Biochemical and microbial studies on kawal, a meat substitute derived by fermentation of Cassia obtusifolia leaves. $J$. Sci. Food Agri. 1985; 36: 881-892.

9. Nuha MO, Isam AMA and EB Elfadil Chemical composition, anti-nutrients and extractable minerals of Sicklepod (Cassia obtusifolia) leaves as influenced by fermentation and cooking. Int. Food Res. J. 2010; 17: 775-785.

10. Algadi MZ and NE Yousif Anti-Nutritional Factors of Green Leaves of Cassia obtusifolia and Kawal. J. Food Process Technol. 2015; 6:9.

11. Mackey AP, Miller EN and WA Palmer Sicklepod in Queensland (Senna obtusifolia). Pest Status Review Series. Land Protection, Department of Natural Resources and Mines, Queensland, 1997: 46.

12. Irwin HS and RC Barneby The American Cassiinae. Memoirs of the New York Botanical Garden. 1982; 5:1-918.

13. Parsons WT and EG Cuthbertson Noxious weeds of Australia. Inkata Press, Melbourne/Sydney, 1992: 692. 


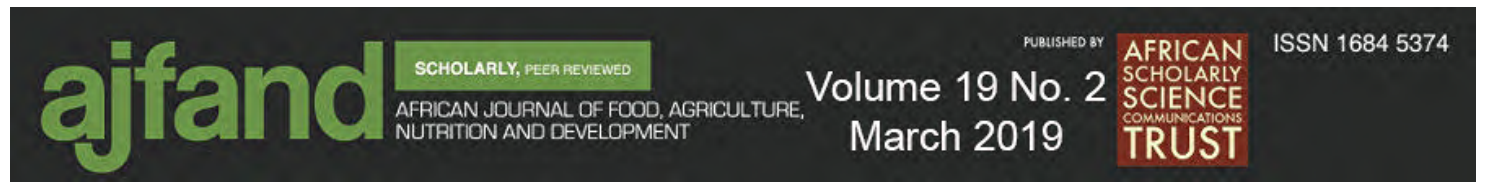

14. PROTA 11 (2) Ressources végétales de l'Afrique tropicale. vol. 11 (2). Plantes médicinales, tome 2. éd. par G.H. Schmelzer\& A. Gurib-Fakim. Wageningen, Fondation PROTA- CTA, 2013: 417.

15. Paul $\mathbf{R}$ and $\mathbf{L}$ Bonne Présentation de deux méthodes originales visant à faciliter dans les IAA, la mise en œuvre des bonnes pratiques d'hygiène et de fabrication ainsi que de la méthode HACCP, telles que de finies par le codex alimentaires. Université de Toulouse, 2013: 1.

16. FAO. Fermented fruits and vegetables. A global perspective. FAO Agricultural services bulletin No. 134. ISBN 92-5-104226-8, Rome, Italy, 1998.

17. Louembé $\mathbf{D}$, Kobawila $\mathbf{S C}$, Bouanga $\mathbf{G}$ and $\mathbf{S}$ Kéléké Etude microbiologique des feuilles fermentées de manioc: "Ntoba Mbodi". Tropicultura. 2003; 21(3): 106-111.

18. Ogbadu $\mathbf{L}$ and RN Okagbue Bacterial fermentation of soya bean for dawadawa production. J. Appl. Bacteriol. 1998; 65: 353-356.

19. Manas RS, Marimuth A, Ramesh CR and PR Rizwana Fermented Fruits and Vegetables of Asia: A Potential Source of Probiotics. Biotechnol. Res. Int.2014; 9.

20. Debajit B, Yadav RNS, Ankush S, Lubana S and KC Anand Production, purification and characterization of natto kinase from Bacillus subtilis, isolated from tea garden soil samples of dibrugarh, assam. Asian J. Pharm Clin. Res. 2012; 5:124-125.

21. Ouoba LII, Diawara B, Amoa-Awua WK, Traore AS and $P$ Lange Møller Genotyping of starter cultures of Bacillus subtilis and Bacillus pumilus for fermentation of African locust bean (Parkia biglobosa) to produce Soumbala. Int. J. Food. Microbiol. 2004; 90: 197-205.

22. Savadogo A, Ilboudo AJ, Gnankiné $O$ and AS Traoré Numeration and Identification of thermotolerant endospore-forming Bacillus from two fermented condiments Bikalga and Soumbala. Adv. Env. Biol. 2011; 5(9): 29602966.

23. Allangheny NO, Banu ZA, Campbell-Platt G and JD Owens Control of ammonia formation during Bacillus fermentation of legumes. Int. J. food Microbiol. 1996; 20: 321-333.

24. Oguntoyinbo FA, Sanni AI, Franz CMAP and WH Holzapfel In-vitro fermentation studies for selection and evaluation of Bacillus strains as starter cultures for production of okpehe, a traditional African fermented condiment. Int. J. Food. Microbiol. 2007; 113: 208-218.

25. Tamang B and JP Tamang Role of lactic acid bacteria and their functional properties in goyang a fermented leafy vegetable product of the Sherpas. J. Hill Res. 2007; 20: 53-61. 


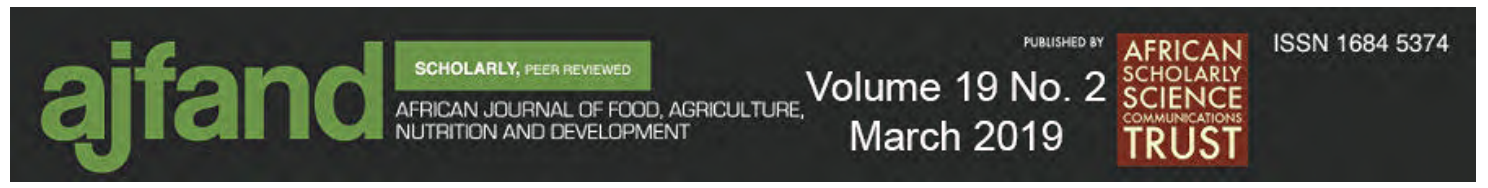

26. Kaboré D, Sawadogo-Lingani H, Dicko MH, Diawara B and M Jakobsen Acid resistance, bile tolerance and antimicrobial properties of dominant LAB isolated from traditional maari, baobab seeds fermented condiment. Afr.J. Biotechnol. 2012; 11(5): 1197-120.

27. Azokpota P, Møller PL, Hounhouigan JD and M Jakobsen Biodiversity of predominant Bacillus isolated from afitin, iru and sonru at different fermentation time. Int. J. Biol. Chem. Sci. 2007; 1(3): 211-222.

28. Jideani AO and CR Okeke Comparative study of microorganisms and sensory attributes of condiments from the fermentation of different seeds. Plant Foods Hum Nutr. 1991; 41(1): 27-34.

29. Dakwa S, Sakyi-Dawson E, Diako C, Takyiwa Annan N and WK AmoaAwua Effect of boiling and roasting on the fermentation of soybeans into dawadawa (soy-dawadawa). Int. J. of Food Microbiol. 2005; 104: 69-82.

30. Paster DL, Woltering A, Nikiema D, Senbeto D, Fatondji $J$ and $J$ Ndjeunga ISHS. Acta Hort. 2007;752: 299-302.

31. Shailesh MK Pharmacognostical studies on the leaves of Cassia obtusifolia Linn. Asian J. Plant Sci. Res. 2012; 4: 421-427.

32. Pasternak DL, Woltering A, Nikiema D, Senbeto D, Fatondji $\mathbf{J}$ and $\mathbf{J}$ Ndjeunga Domestication of Sennaobtusifolia, an important leafy vegetable for the Sahel. ISHS ActaHorticulturae 752:International Conference on Indigenous Vegetables and Legumes. Prospectus for Fighting Poverty, Hunger and Malnutrition, 2007.

33. Becker B Wild plants for human nutrition in the Sahelian Zone. J. Arid Env. 1986; 1: 61-64.

34. Dirar A Kawal, Meat substituent from fermented Cassia obtusifolia leaves. Econ. botan. 1984; 38: 342-349.

35. Adjoudji $\mathbf{O}$, Ngassoum $\mathbf{M}$ and $\mathbf{C}$ Kamga Chemical composition of Cassia obtusifolia Leaves. J. Food. Technol. 2010; 3(3): 453-455.

36. Abakar IL, Savadogo A, Tapsoba F, Guira F and Y Traore Identification and Biochemical Analysis of Microorganisms involved in the Fermentation of KawalA Traditional Fermented Sicklepod Leaves (Senna obtusifolia). Int. J. Curr. Microbiol. Appl Sci. 2016; 9: 261-270.

37. Mbaiguinam M, Mahamout Y, Mianpereum T and D Bernard Constituents of Kawal, fermented Cassia obtusifolia leaves, a traditional food from Chad. Afr. J. Biotechnol. 2005; 4:1080-1083. 